\title{
Variação temporal de larvas de Chironomidae (Diptera) no Alto Rio Paraguai (Cáceres, Mato Grosso, Brasil
}

\author{
Fernando H. Aburaya \& Claudia T. Callil
}

\begin{abstract}
Laboratório de Ecologia Animal, Departamento de Biologia e Zoologia, Instituto de Biociências, Universidade Federal de Mato Grosso. Avenida Fernando Corrêa da Costa, 78000-900 Cuiabá, Mato Grosso, Brasil.

E-mail: aburayafh@yahoo.com.br; callil@ufmt.br
\end{abstract}

\begin{abstract}
Temporal variation of Chironomidae larvae (Diptera) in the Upper Paraguay River (Cáceres, Mato Grosso, Brazil. The aim of this study is to determine the influence of environmental variables on the time distribution of assemblages of Chironomidae in the Upper Paraguay River. The samples were collected from November 2004 to October of 2005. To consider the diversity of habitats in the region, nine different sampling points were sampled and 2988 individuals were collected, distributed in 34 species, eight tribes and three subfamilies. The genus Labrundinia sp.1, Polypedilum (Asheum) and Polypedilum (Tripodura) occurred almost every month during the study. Richness and abundance were higher during the dry period of the year, and the genus Polypedilum was the most abundant. There was evident the effect of the environmental variables on the attributes of diversity in the Upper Paraguay River.
\end{abstract}

KEY WORDS. Pantanal; sazonal distribution; structure community; richness; abundance.

RESUMO. A composição das assembléias de Chironomidae e os possíveis fatores ambientais que influenciam a distribuição temporal deste grupo no alto rio Paraguai, em Cáceres, foram abordados no presente estudo durante o período de novembro de 2004 a outubro de 2005. Para considerar a diversidade de habitats na região, selecionamos nove pontos amostrais, onde coletamos 2988 indivíduos, distribuídos em 34 espécies pertencentes a oito tribos de três subfamílias. Os gêneros Labrundinia sp.l, Polypedilum (Asheum) e Polypedilum (Tripodura) estiveram presentes em quase todos os meses amostrados. A maior riqueza e abundância foram registradas no período de seca, revelando Polypedilum como o gênero mais expressivo. Ficou evidente o efeito das vaiáveis ambientais sobre os atributos da diversidade do alto rio Paraguai.

PALAVRAS-CHAVE. Pantanal; distribuição sazonal; estrutura da comunidade; riqueza; abundância.

Os rios comportam-se como sistemas de fluxo contínuo unidirecional, nos quais os diferentes habitats apresentam características peculiares em cada período hidrológico (WARD et al. 2002). Nesses ambientes as comunidades aquáticas podem ser controladas direta ou indiretamente por fatores abióticos e bióticos (Bueno et al. 2003).

A distribuição dessas comunidades, principalmente os invertebrados bentônicos, está diretamente ligada a fatores limnológicos (GALDEAN et al. 2000, BAPTISTA et al. 2001) como: o nível fluviométrico (TAKEDA et al. 1997), a composição do sedimento (Príncipe \& Corigliano 2006), o recurso alimentar disponível (SANSEVERINO et al. 1998) e também às interações tróficas interespecíficas (WALKER 1998).

A fauna bentônica apresenta um papel funcional importante nos ecossistemas límnicos, por participar no processo de transformação da matéria orgânica particulada (DEvINE \& VANNI 2002) e servir de alimento para níveis tróficos adjacentes e superiores (Callisto \& Esteves 1998, Moreira \& Zuanon 2002).
Os insetos aquáticos são frequentemente utilizados como ferramenta na compreensão das alterações ambientais (TAKEDA et al. 1997, FonseCA-GesSNer \& Guereshi 2000). Através deles, podemos obter registros dos processos ecológicos decorrentes das mudanças ocasionadas pelas variações hidrológicas. Portanto, o estudo destes organismos fornece informações valiosas para o entendimento da estrutura e funcionamento das comunidades límnicas (Moulton 1998).

Chironomidae constitui um grupo importante sob o ponto de vista ecológico. O fato de apresentarem ampla distribuição na maioria dos ecossistemas aquáticos e ocorrer em alta densidade e riqueza (Hirabayashi \& WotTon 1998), além de possuírem hábitos sedentários, capacita este grupo a refletir as mudanças ecológicas locais (Callisto \& Esteves 1998, GoularT \& CAllisto 2003).

Assembléias de Chironomidae sofrem alterações sazonais significativas, aumentando suas densidades durante o período de estiagem. Evento observado em lagoas (Ввоокs 2000, Li et al. 
2002), rios (MARques et al. 1999) e córregos (KiKUChi \& Uieda 1998, Robinson et al. 2001). As variações espaciais e temporais desses organismos têm sido demonstradas para a planície de inundação do alto rio Paraná (TAKEDA et al. 1991) e para a planície de inundação do rio Paraguai (MARChese et al. 2005).

Na região do Pantanal, as mudanças das características limnológicas são decorrentes do pulso de inundação (JuNK et al. 1989). Os trabalhos de JunK \& DA Silva (1995) e DA Silva et al. (2001) explicam que tais alterações ocorrem de maneira periódica e sistematizada. Porém, estudos sobre identificação e monitoramento dos efeitos destas alterações sobre as larvas de Chironomidae para a região estão restritos aos trabalhos de Ezcurra de Drago et al. (2004) e Marchese et al. (2005) para o rio Paraguai.

O objetivo deste estudo foi avaliar a composição e estrutura das assembléias de Chironomidae e avaliar quais fatores abióticos influenciam a distribuição temporal, no Alto Paraguai, município de Cáceres, Mato Grosso.

\section{MATERIAL E MÉTODOS}

\section{Área de estudo}

A área de estudo localiza-se no Município de Cáceres, Mato Grosso $\left(16^{\circ} 11^{\prime} 42^{\prime \prime} \mathrm{S}\right.$ e $\left.57^{\circ} 40^{\prime} 51^{\prime \prime} \mathrm{W}\right)$, à margem esquerda do rio Paraguai, a qual apresenta altitude de $158 \mathrm{~m}$ (Ferreira 2001). O rio Paraguai nasce na Chapada do Parecis, numa área de importância hidrográfica sul-americana, pois reúne além da nascente do rio Paraguai, tributários do rio Amazonas (РСвAP 1997). De acordo com a classificação de Köppen, a Bacia do Alto Paraguai, apresenta Clima Tropical de Savana (AW), sendo a oscilação mínima e máxima entre $17-20^{\circ} \mathrm{C}$ (РСвAP 1997). A precipitação pluviométrica da região é marcada por duas estações bem definidas: chuvosa entre os meses de novembro a abril, com inundações nas partes baixas; e outra seca bem definida no restante dos meses. Os índices pluviométricos apresentam três períodos distintos ao longo do ano, alcançando os maiores valores entre dezembro e março, com precipitações médias mensais de 205,4 mm. O período de seca, apresenta precipitações médias mensais variando de 65,8 a $105,5 \mathrm{~mm}$, sendo os meses de junho, julho e agosto os mais secos.

Com a proposta de representar a diversidade de habitats existentes no local, foram selecionados e amostrados nove pontos com características diferentes. Todos localizados nos limites da área urbana de Cáceres, entre a Baía do Malheiros $\left(16^{\circ} 03^{\prime} 34,38^{\prime \prime}\right.$ S e $\left.57^{\circ} 41^{\prime} 27,00^{\prime \prime} \mathrm{W}\right)$ e poção do Roda Barbado $\left(16^{\circ} 06^{\prime} 50,91^{\prime \prime}\right.$ S e $\left.57^{\circ} 43^{\prime} 44,85^{\prime \prime} \mathrm{W}\right)$. Para as análises referentes à variação temporal, consideraram-se os nove pontos como uma amostra composta, descartando a possibilidade de correlação espacial entre os pontos.

\section{Procedimento de campo}

Para caracterização limnológica dos pontos amostrados, foram medidos em campo os valores do potencial hidrogeniônico - pH (100 YSI), temperatura - T ( $\left.{ }^{\circ} \mathrm{C}\right)$ (100 YSI), condutividade elétrica - Cond. $\left(\mu \mathrm{S} . \mathrm{cm}^{-1}\right)($ OREON 1150A+), oxigênio dissolvido - OD (mg.1-1 $)$ (200WSI), transparência da água - Trans. (cm) (disco de Secchi) e profundidade do rio - Prof. (m) (régua limnética). Tréplicas de amostras de água foram coletadas e preservadas com ácido nítrico para análise de nitrogênio total (NKT) e fósforo total (PT) (AlLen 1989), utilizando o método fenato alcalino e uma amostra não preservada para análise de ortofosfato (método colorimétrico) (APHA 1998). Todo o material foi transportado em frascos plásticos (1 L) e acondicionado em caixas térmicas resfriadas. As análises foram feitas no Laboratório de Monitoramento Ambiental da Secretaria de Meio Ambiente (SEMA-MT).

Para a obtenção do material em suspensão, filtros de fibra de vidro (Watman $\mathrm{GF} / \mathrm{C}-47 \mathrm{~mm}$ ) foram calcinados a $550^{\circ} \mathrm{C}$ para a filtragem de $200 \mathrm{ml}$ de água e posterior secagem em estufa a $105^{\circ} \mathrm{C}$. O teor de matéria orgânica em suspensão foi calculado pela diferença de peso dos filtros antes e depois da filtragem. Em seguida os filtros foram colocados em mufla a $550^{\circ} \mathrm{C}$, onde a diferença dos pesos, consideramos como matéria orgânica em suspensão.

As amostras para o exame bacteriológico foram acondicionas em sacolas específicas, contendo pastilhas de Cloreto e resfriadas a $8^{\circ} \mathrm{C}$. Geralmente são utilizados como indicadores de contaminação fecal Escherichia coli, por fazerem parte do mesmo grupo de bactérias assim denominada. A quantificação de coliformes totais e fecais foi obtida pelo método do substrato definitivo, Collilert (WB-020).

Concomitante às analises limnológicas, em cada ponto amostras de sedimento foram coletadas com pegador de fundo, tipo Petersen modificado $\left(0,0416 \mathrm{~m}^{2}\right)$. Das amostras de sedimento, três destinaram-se à análise biológica e uma para análise de matéria orgânica. Em campo o material coletado foi acondicionado em sacolas plásticas, com suas devidas identificações.

Em seguida, o material foi lavado e previamente triado em um conjunto de peneiras com malhas de 2,0 $\mathrm{mm}, 1,0 \mathrm{~mm}$ e $0,25 \mathrm{~mm}$. O restante do material retido na malha $0,25 \mathrm{~mm}$ foi fixado em álcool 70\% para posterior triagem em laboratório. Com auxílio de microscópio estereoscópico os organismos foram separados em morfoespécies e montadas lâminas, procedimento necessário para identificação das larvas. Os indivíduos pertencencentes à familia Chironomidae foram identificados ao menor nível taxonômico possível, de acordo com TrivinhoStrixino \& Strixino (1995), Merritt \& Cummins (1996), Epler (2001) e Fernández \& Dominguez (2001). Os espécimes testemunhos estão depositados na coleção de invertebrados aquáticos do Laboratório de Ecologia Animal da Universidade Federal de Mato Grosso.

\section{Análise de dados}

A estimativa da densidade de espécies foi feita a partir da área do amostrador e a partir de então, elaborada uma matriz de presença ou ausência dos táxons, simbolizada pelo número de ocorrência nos 12 meses amostrados. Foi estimada a riqueza para cada mês utilizando o método de Jackknife (KreBs 1989). 
De todas as variáveis analisadas, foram utilizadas apenas as que não apresentaram correlação. Os valores referentes às variáveis limnológicas foram padronizados através da divisão de cada variável pela média. Para a ordenação dos meses, aplicou-se uma Análise de Componentes Principais (PCA) utilizando a distância Euclidiana (SYSTAT versão 11.0). também foi aplicada uma ordenação pelo método de escalonamento multidimensional não métrico (NMDS) com o auxílio do programa PcORD (versão 4.0) aonde utilizamos os dados de densidade das espécies nos meses amostrados, resumindo as variáveis em dois eixos. Após realizou-se uma análise de regressão múltipla multivariada entre os dois eixos da NMDS com as variáveis $\mathrm{pH}$, temperatura, condutividade, $\mathrm{OD}$, nível de água.

\section{RESULTADOS}

A tabela I apresenta os valores mínimos e máximos, média e desvio padrão das variáveis consideradas. Profundidade e temperatura variaram ao longo do ano apresentando menores valores no período da seca e maiores no período de cheia. A transparência e condutividade obtiveram valores inversos, com diminuição no período de cheia. O mesmo comportamento foi observado para pH e nitrogênio total (NKT), com menores valores encontrados no período de seca e maiores nos períodos de cheia.

Os valores de material em suspensão, matéria orgânica em suspensão, coliformes totais, fecais, oxigênio dissolvido, PT e DBO (Demanda Bioquímica de Oxigênio) foram marcados com oscilações intercaladas entre os meses amostrados (Fig. 1). Sendo que diferenças significativas foram detectadas (ANOVA $\mathrm{p}<0,05)$ entre transparência, DBO, nutrientes da água, materi- al em suspensão, coliformes (totais e fecais) e profundidade.

Através da PCA, 78\% da alteração contida nas variáveis abióticas foram resumidas em dois eixos. O eixo 1 explicou $56 \%$ de variação relacionada ao nível de água $(0,956)$, pH $(-0,881)$, oxigênio dissolvido $(-0,697)$, condutividade $(0,291)$ e temperatura $(0,721)$. Para o eixo 2 , as variáveis foram resumidas em $22 \%$ representadas pelo nível de água $(0,039), \mathrm{pH}(-0,881)$, oxigênio dissolvido $(0,288)$, condutividade $(-0,918)$ e temperatura $(0,132)$. Na figura 2, elaborada a partir dos eixos obtidos através da PCA nota-se um agrupamento dos meses que compreendem o período de cheia (janeiro a abril) representado no grupo 1; pelos meses intermediários aos períodos de seca e cheia representados no grupo 2 e um terceiro grupo formado pelo mês de setembro, pico da seca.As análises de regressão múltipla multivariada não indicaram haver qualquer influência destas sobre a fauna de Chironomidae.

No total foram identificadas 34 morfo-espécies de Chironomidae, distribuídos em oito tribos e três subfamílias, representando 2988 indivíduos coletados no período de novembro/ 2004 a outubro/2005 (Tab. II). Os valores de riqueza oscilaram ao longo do ano (Fig. 3), sendo observado um aumento do número de espécies durante o período de seca, entretanto não apresentaram diferenças significativas, quando analisadas pelo teste de $\chi^{2}(\mathrm{p}=0.94)$. O mês de maio apresentou menor riqueza com a ocorrência de quatro espécies, enquanto o mês de julho apresentou maior riqueza com 35 espécies.

Os gêneros Labrundinia sp.1, Polypedilum (Asheum) e Polypedilum (Tripodura) apresentaram maiores freqüências de ocorrência, estando presente em quase todos os meses amostrados (92\%). Enquanto que Brundiniella, Fissimentum desiccatum,

Tabela I. Valores mínimos, máximos, média anual e desvios padrões (DP) das variáveis limnológicas analisadas no alto rio Paraguai. Cáceres, Mato Grosso.

\begin{tabular}{|c|c|c|c|}
\hline Meses & Mínimo & Máximo & Média \pm DP \\
\hline $\mathrm{pH}$ & 5,20 & 8,20 & $6,600 \pm 0,700$ \\
\hline Oxigênio dissolvido $\left(\mathrm{mg} \mathrm{L}^{-1}\right)$ & 2,72 & 12,80 & $7,300 \pm 2,060$ \\
\hline Condutividade ( $\mu$ S.cm-1) & 28,00 & 101,00 & $39,000 \pm 9,000$ \\
\hline Temperatura $\left({ }^{\circ} \mathrm{C}\right)$ & 23,00 & 33,90 & $28,000 \pm 2,000$ \\
\hline Demanda bioquímica de oxigênio $\left(\mathrm{mg} \cdot \mathrm{L}^{-1}\right)$ & 0,010 & 1,35 & $0,670 \pm 0,300$ \\
\hline Nitrogênio total $\left(\mathrm{mg} \cdot \mathrm{L}^{-1}\right)$ & 0,001 & 1,42 & $0,170 \pm 0,190$ \\
\hline Fósforo total (mg. $\left.\mathrm{L}^{-1}\right)$ & 0,150 & 0,85 & $0,100 \pm 0,050$ \\
\hline Ortofosfato (mg. $\left.\mathrm{L}^{-1}\right)$ & 0,020 & 0,24 & $0,042 \pm 0,037$ \\
\hline Matéria suspensa (mg. $\left.\mathrm{L}^{-1}\right)$ & 0,600 & 0,48 & $0,190 \pm 0,200$ \\
\hline Matéria orgânica suspensa (mg. $\left.\mathrm{L}^{-1}\right)$ & 0,060 & 0,15 & $0,900 \pm 0,370$ \\
\hline Material orgânico sedimentado (\%) & 1,740 & 2,47 & $2,210 \pm 0.350$ \\
\hline Coliformes Total (NMP/ml) & 24980 & 91 & $1854,000 \pm 4198$ \\
\hline Coliformes Fecais ( NMP/ml) & 389 & 1,00 & $205,000 \pm 5810$ \\
\hline Profundidade (m) & 0,400 & 7,70 & $2,820 \pm 1,660$ \\
\hline Nível d'água (m) & 1,180 & 4,68 & $2,270 \pm 1,190$ \\
\hline Transparência (cm) & 95 & 19,00 & $47,000 \pm 20,20$ \\
\hline
\end{tabular}



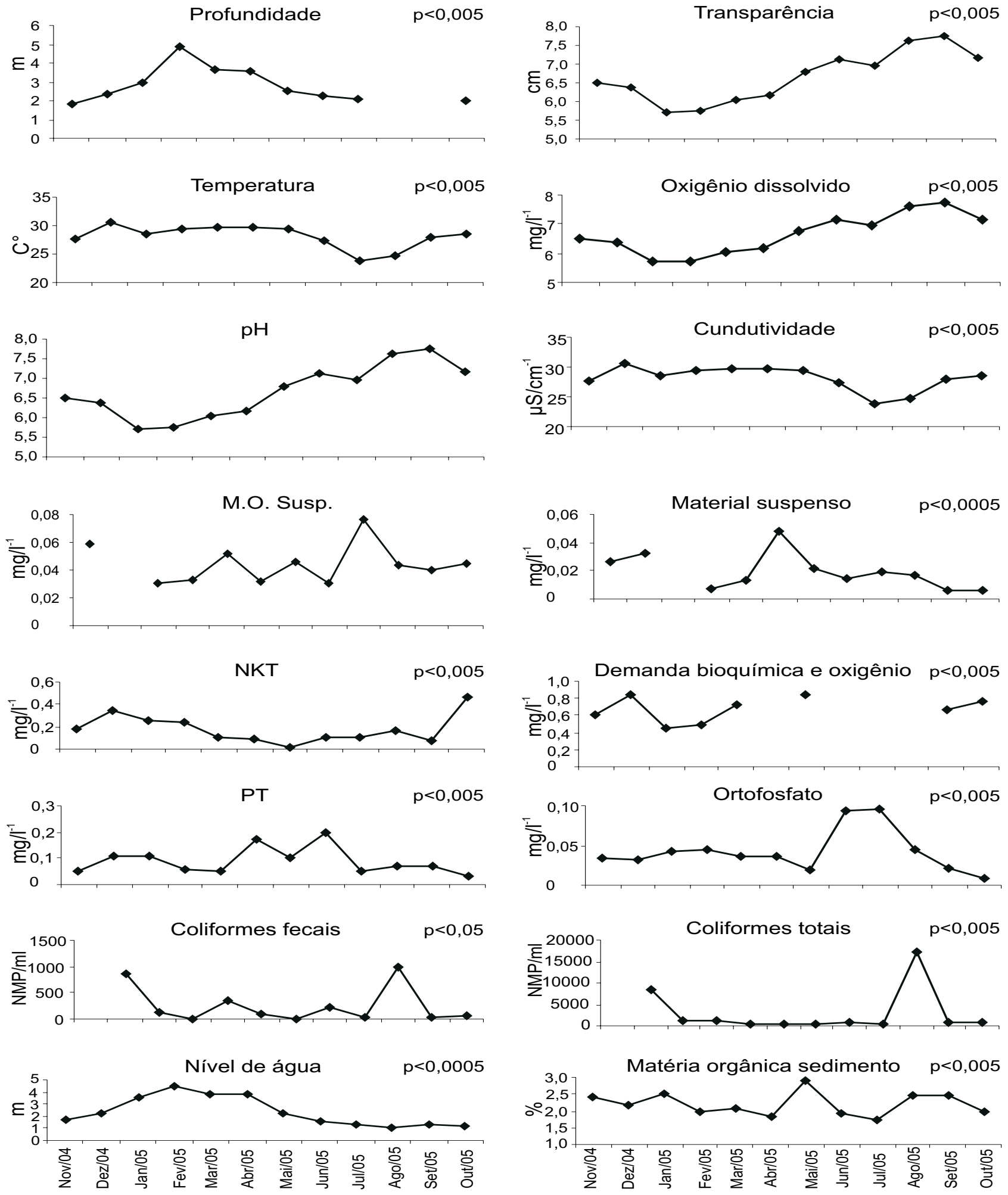

Figura 1. Variação mensal dos parâmetros físicos e químicos nos pontos de coleta no alto rio Paraguai, com respectivos valores de " $p^{\text {" }}$ (Kruskal-Wallis), Cáceres, Mato Grosso.

Revista Brasileira de Zoologia 24 (3): 565-572, setembro 2007 


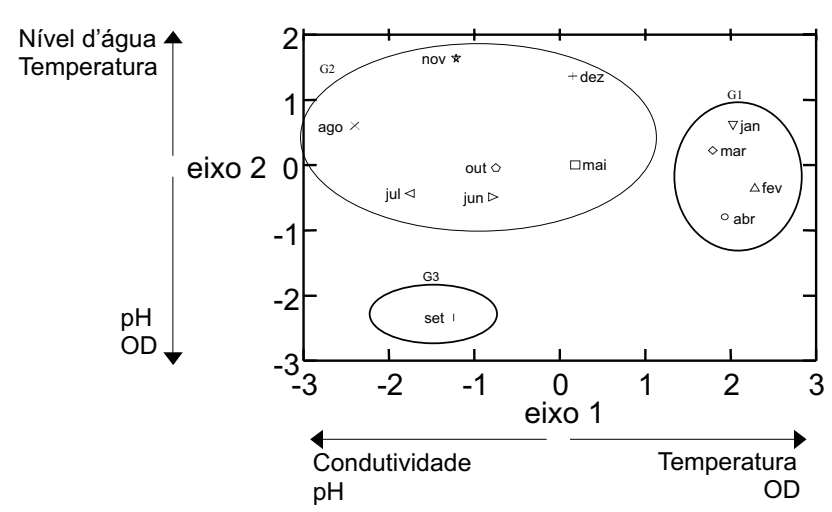

Figura 2. Análise de Componente Principais (PCA), relacionando os variáveis limnológicas $(\mathrm{pH}$, temperatura, condutividade, OD e nível d'água), para os 12 meses no alto rio Paraguai, município de Cáceres, Mato Grosso.

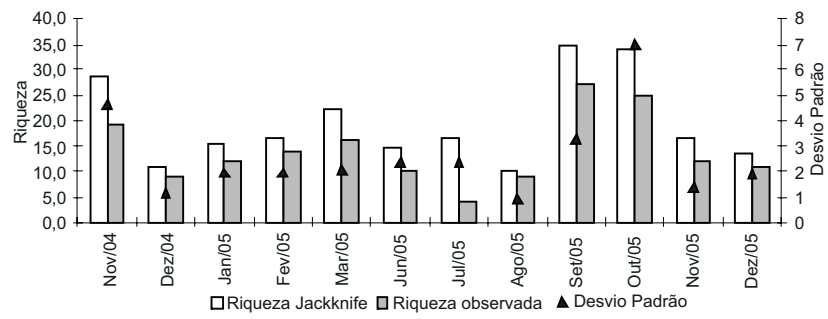

Figura 3. Riqueza estimada (Jackknife), desvios padrões e riqueza observada de Chironomidae ao longo de um período sazonal, no rio Paraguai, Cáceres, Mato Grosso.

Aedokritus, Rheotanytarsus, Pseudochironomus e Paralauterborniella foram as espécies de menor ocorrência (8\%), estando presentes apenas nos meses de julho e agosto.

Durante a seca, houve um expressivo aumento da densidade total das larvas de Chironomidae, passando de aproximadamente 180 ind. $\mathrm{m}^{-2}$ (maio) para 840 ind.m ${ }^{2}$ (agosto) (Fig. 4). Este está relacionado a Polypedilum (Asheum) (45,8\%), Polypedilum (Tripodura) (11,5\%), Labrundinia sp.1 (11,4\%), Polypedilum gr. fallax $(4,22 \%)$ e Tanytarsus $(2,68 \%)$, espécies de maiores densidades numéricas dentre todas as encontradas.

\section{DISCUSSÃO}

No Pantanal, de uma maneira geral, as flutuações das variáveis físicas, químicas e biológicas são mantidas pelo pulso de inundação periódico na ATTZ - zona de transição aquática terrestre (Junk et al. 1989). Conforme descrito por DA Silva et al. (2001), o aporte de matéria orgânica proveniente da ATTZ para a calha do rio, promove um aumento dos nutrientes dissolvidos na água. DA Silva \& Esteves (1995) relatam que as concentrações de ortofosfato, fósforo total e nitrogênio total na água passam a ser maiores no período de seca. Tal situação acarreta alterações

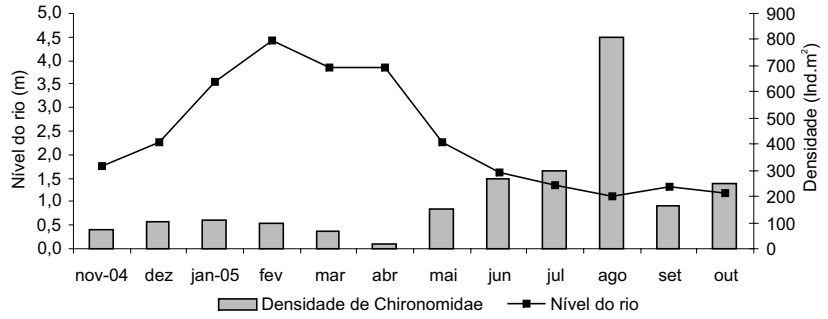

Figura 4. Período hidrológico no alto rio Paraguai, Cáceres, Mato Grosso, com as medidas do nível do rio e densidades de Chironomidae.

nos valores de $\mathrm{pH}$, condutividade, oxigênio dissolvido, entre outras (Esteves 1998, AmÂNCIO et al. 2004), interferindo diretamente nas condições ambientais disponíveis para os organismos.

No rio Paraguai foi registrado grande número de espécies de larvas de Chironomidae nos meses amostrados. MARCHESE et al. (2005) no mesmo rio verificaram a ocorrência de 20 espécies de larvas de Chironomidae, enquanto que neste trabalho o maior valor de riqueza chegou a 34 espécies durante os meses de seca. Este fato demonstra a necessidade de considerar a variação sazonal em trabalhos que visem ao inventário desse grupo taxonômico. Conforme OtT \& Carvalho (2001) há eficiência no método de amostragem quando os valores de riqueza estimada e riqueza observada são semelhantes. No presente estudo, foi encontrada a mesma situação, onde os índices de riquezas observada e estimada pelo Jackknife, não apresentaram diferenças significativas, fato comprovado pelo teste de comparação entre amostras. Segundo WALKER (1998) além dos fatores abióticos descritos anteriormente, interações biológicas diretas e indiretas possivelmente também influenciam a distribuição da assembléia de Chironomidae.

Takeda et al. (1997), Walker (1998), Fonseca-Gessner \& Guereschi (2000) e Higuti \& TAKEDA (2002) em seus trabalhos em ambientes lóticos, relatam diminuição das densidades numéricas de Chironomidae no período de cheia, como resultado do aumento da velocidade de correnteza e desestruturação do sedimento. No período de águas baixas há uma reestruturação dos habitats, favorecendo a permanência dos organismos bentônicos. No rio Paraguai quando as águas começaram a baixar, a densidade de Chironomidae aumentou, provavelmente devido à maior diversificação de habitats.

Em regiões temperadas, Oliver \& Dillon (1997) e LenCioni \& Rossano (2005) descreveram a dominância de Orthocladiinae sobre Chironominae, assim como em nosso estudo, SERRAno et al. (1998) em regiões tropicais encontraram o inverso, com dominância da Chironominae sobre Orthocladiinae, ressaltando a preferência de Chironominae por ambientes de clima tropical.

No rio Paraguai, a maior densidade encontrada para Chironominae, esteve relacionada com as larvas de Polypedilum, normalmente citado por ocorrer em altas densidades (SchмiD 
Tabela II.Densidade relativa de Chironomidae observada durante novembro de 2004 a outubro de 2005 no rio Paraguai, Cáceres, Mato Grosso.

\begin{tabular}{|c|c|c|c|c|c|c|c|c|c|c|c|c|}
\hline & Jan-05 & Fev & Mar & Abr & Mai & Jun & Jul & Ago & Set & Out & Nov-05 & Dez \\
\hline \multicolumn{13}{|l|}{ Tanypodinae } \\
\hline \multicolumn{13}{|l|}{ Macropelopiini } \\
\hline Brundiniella Roback, 1978 & & & & & & & 1 & & & & & \\
\hline \multicolumn{13}{|l|}{ Pentaneurini } \\
\hline Ablabesmyia Johannsen, 1905 & & 2 & & & & & 1 & 1 & 1 & & 1 & \\
\hline Denopelopia Roback \& Rutter, 1988 & & & 1 & 1 & 1 & 2 & 1 & 1 & 1 & 1 & 1 & 2 \\
\hline Labrundinia sp. 1 & 1 & 1 & 1 & 1 & 1 & 3 & 2 & 2 & & 2 & & 1 \\
\hline Labrundinia sp. 2 & 2 & & & & 1 & 1 & & 1 & & 1 & 1 & 1 \\
\hline Pentaneura Philippi, 1865 & & & & & & & 1 & 1 & & & & \\
\hline Thienemannimyia Fittkau, 1957 & & 1 & & & & & 1 & & & & & \\
\hline \multicolumn{13}{|l|}{ Procladiini } \\
\hline Djalmabatista pulcher Johannsen 1908 & 1 & 1 & 1 & & 1 & 1 & 1 & & & & 1 & \\
\hline Procladius Skuse, 1889 & 2 & 1 & & & 1 & & 2 & 1 & & & 1 & \\
\hline \multicolumn{13}{|l|}{ Coelotanypodini } \\
\hline Coelotanypus Kieffer, 1913 & 1 & & 1 & & & & & 1 & & 2 & 1 & \\
\hline \multicolumn{13}{|l|}{ Chironominae } \\
\hline \multicolumn{13}{|l|}{ Chironomini } \\
\hline Chinonomini & 1 & & 1 & & 1 & & & 1 & 1 & & & \\
\hline Polypedilum (Asheum) Sublette \& Sublette, 1983 & 2 & & 2 & 1 & 3 & 3 & 2 & 3 & 2 & 3 & 2 & 2 \\
\hline Polypedilum (Polypedilum) Kieffer, 1912 & & & & & & & 1 & & & & 1 & \\
\hline Polypedilum gr. fallax Johannsen, 1905 & & 1 & 1 & & 1 & 1 & 2 & 1 & 1 & 2 & 1 & 1 \\
\hline Polypedilum (Tripodura) & 2 & 2 & 1 & 1 & 2 & 2 & 2 & 2 & 2 & 2 & & 1 \\
\hline Axarus Roback, 1980 & & & & & & & 2 & 1 & & & & \\
\hline Cryptochironomus Kieffer, 1918 & & 1 & & & & & 1 & 1 & & & 2 & \\
\hline Goeldichironomus Fittkau, 1965 & & & 1 & 1 & 1 & & 1 & 1 & 1 & & 1 & 1 \\
\hline Harnischia Kieffer, 1921 & & & 1 & 1 & & & 2 & 1 & 2 & 1 & 1 & \\
\hline Chironomus gr. salinarius Thienemann, 1915 & & 2 & & & & & 1 & 1 & & & 1 & \\
\hline Paratendipes Kieffer, 1911 & 1 & & 1 & 1 & 1 & 1 & 1 & 2 & 2 & 2 & & 1 \\
\hline Paralauterborniella Lenz, 1941 & & & & & & & & 1 & & & & \\
\hline Tribelos Townes, 1945 & 1 & 1 & & & & & 2 & & & & 1 & \\
\hline Fissimentum desiccatum Cranston \& Nolte, 1996 & & & & & & & 2 & & & & & \\
\hline Aedokritus Roback, 1958 & & & & & & & 1 & & & & & \\
\hline \multicolumn{13}{|l|}{ Tanytarsini } \\
\hline Caladomyia ortoni Säwedall, 1981 & & & & & 1 & & 1 & 2 & & 1 & & \\
\hline Tanytarsus Van del Wulp, 1874 & 2 & 1 & 1 & 1 & & & 1 & 2 & 2 & 1 & 1 & \\
\hline Tanytarsus rhabdomantis Trivinho-Strixino \& Strixino, 1991 & & 1 & 1 & & & & 1 & & & & 1 & \\
\hline Rheotanytarsus Thienemann \& Bause in Bause, 1913 & & & & & & & & 1 & & & & \\
\hline Tanytarsini gen. C Trivinho-Strixino \&. Strixino, 1995 & & & & & & & 1 & 1 & & & 1 & \\
\hline Tanytarsini gen. A Trivinho-Strixino \&. Strixino, 1995 & & & 1 & & & & 1 & 1 & 1 & & & \\
\hline \multicolumn{13}{|l|}{ Pseudochironomini } \\
\hline Pseudochironomus Sæther, 1977 & & & & & & & & 1 & & & & \\
\hline \multicolumn{13}{|l|}{ Orthocladiinae } \\
\hline \multicolumn{13}{|l|}{ Corynoneurini } \\
\hline Corynoneura Winnertz, 1846 & 1 & 1 & 1 & 1 & 1 & 1 & & & 1 & & & \\
\hline Thienemanniella Kieffer, 1911 & & 1 & 1 & 1 & & & 1 & & 1 & & 1 & 1 \\
\hline
\end{tabular}

1) Densidades entre 1 e 100 indivíduos; 2) densidades entre 101 e 1000 indivíduos; 3) densidades entre 1001 e 10000 indivíduos. 
1992, Marchese et al. 2005, Trivinho-STrixino \& Strixino 2005) e tido como um gênero cosmopolita, representado por numerosas espécies e reportado em vários trabalhos de Oliver \& Dillon (1997), Serrano et al. (1998), Sanseverino et al. (1998), Bueno (2003) e MARChese et al. (2005).

As considerações aqui apresentadas demonstram mais uma vez que o regime hidrológico altera as características físicas, químicas e biológicas. Ficou evidente o efeito destas variações sobre os atributos da diversidade do alto rio Paraguai. Vale destacar a importância do conhecimento das espécies de Chironomidae, pelo fato de se tratar de organismos "resposta" das condições e alterações sofridas nos habitats. Além disso, o conhecimento da riqueza e distribuição das espécies encontradas, torna se uma importante fonte referenciada para planos de manejo e conservação de recursos aquáticos.

\section{AGRADECIMENTOS}

Ao Programa de Pós-Graduação em Ecologia e Conservação da Biodiversidade (IB, UFMT), ao Núcleo Estudos Ecológicos do Pantanal; à SEMA/MT - Laboratório de Monitoramento Ambiental da Secretaria de Meio Ambiente; à FAPEMAT processo 4.2.2.82/02-2004-E e ao Departamento de Ciências Biológicas (UNEMAT, Cáceres). Aos colegas do Laboratório de Ecologia Animal (UFMT). Este trabalho fez parte da dissertação de mestrado desenvolvido com auxílio CAPES processo 5001019003PO.

\section{REFERÊNCIAS BIBLIOGRÁFICAS}

AlLen, S.E. 1989. Chemical analysis of ecological materials. Oxford, Blackwell Scientific Publications, $2^{\text {nd }}$ ed., 368p.

AmÂncio, A.L.L.; W.R.L. Farias; A.R.S. Neto \& A.S. Lopes. 2004. Physical-chemicals parameters characterization of the Pereira de Miranda dam. Revista Ciência Agronômica 35 (2): 340-348.

АРнA. 1998. Standart methods for the examination of water and wastewater. Baltimore, American Public Health Association, AWWA, WEF, $2^{\text {nd }}$ ed. 1220p.

Baptista, D.F.; L.F.M. Dorvillé; D.F. Buss \& J.L. Nessimian. 2001. Spatial and temporal organization of aquatic insect's assemblages in the longitudinal gradient of a tropical river. Revista Brasileira de Biologia 61 (2): 295-304.

Bвоокs, R.T. 2000. Annual and seasonal variation and the effects of hydroperiod on benthic macroinvertebrates of seasonal forest (vernal) ponds in central Massachusetts, USA. Wetlands 20 (4): 707-715.

Bueno, A.P.; G. Bond-Buckup \& B.D.P. Ferreira. 2003. Estrutura da comunidade de invertebrados bentônicos em dois cursos d'água do Rio Grande do Sul, Brasil. Revista Brasileira de Zoologia 20 (1): 115-125.

Callisto, M. \& F.A. Esteves. 1998. Biomonitoramento da macrofauna bentônica de Chironomidae (Diptera) em dois igarapés amazônicos sob influência das atividades de uma mineração de bauxita, p. 299-309. In: J.L. Nessimian \& A.L.
Carvalho (Eds). Ecologia de insetos aquáticos. Rio de Janeiro, PPGE-UFRJ, Série Oecologia Brasiliensis, I+309p.

DA Silva, C.J. \& F.A. Esteves. 1995. Dinâmica das características limnológicas das baías Porto de Fora e Acurizal (Pantanal de Mato Grosso) em função da variação do nível da água, p. 47-60. In: F.A. Esteves (Ed.). Estrutura, funcionamento e manejo de ecossistemas brasileiros. Rio de Janeiro, PPGEUFRJ, Série Oecologia Brasiliensis, I+597p.

da Silva, C.J.; K.M. Wantzen; C.N. da Cunha \& F.A. Machado. 2001. Biodiversity in the Pantanal wetland, Brasil, p. 187215. In: B. Gopal; W.J. JunK \& J.A. Davis (Eds). Biodiversity in wetlands: assessment function and conservation. Leiden, Backhuys Publishers, II+353p.

Devine, J.A. \& M.J. VAnni. 2002. Spatial and seasonal variation in nutrient excretion by benthic invertebrates in a eutrophic reservoir. Freshwater Biology 47 (1): 1107-1121.

EPLER, J.H. 2001. Identification manual for the larval Chironomidae (Diptera) of North and South Corolina. Orlando, Departament of Enviromental and Natural Resources, 495p.

Esteves, F.A. 1998. Fundamentos de Limnologia. Rio de Janeiro, Interciência, $2^{\text {nd }}$ ed., $602 \mathrm{p}$.

Ezcurra de Drago, I.; M. Marchese \& K.M. Wantzen. 2004. Benthos of a large neotropical river: spatial patterns and species assemblages in the Lower Paraguay and its floodplains. Archiv für Hydrobiologie 160 (3): 347-374.

Fernández, H.R. \& E. Domingues. 2001. Guia para la determinación de los artrópodos bentônicos sudamericanos. Tucumán, Universidad Nacional de Tucumán, 282p.

Ferreira, J.C.V. 2001. Mato Grosso e seus municípios. Cuiabá, Secretaria de Estado da Cultura, 668p.

FONSECA-GESSNER, A.A. \& R.M. GUERESCHI. 2000. Macroinvertebrados bentônicos na avaliação da qualidade da água de três córregos na Estação Ecológica de Jataí, Luiz Antônio, SP, Brasil, p. 707719. In: J.E. SANTOS \& S.R. PIREs. (Eds). Estudos integrados em ecossistemas: Estação Ecológica de Jataí. São Carlos, Editora da Universidade Federal de São Carlos, 720p.

Galdean, N.; M. Callisto \& F.A.R. Barbosa. 2000. Lotic ecosystems of Serra do Cipó, southeast Brazil: water quality and a tentative classification based on the benthic macroinvertebrate community. Aquatic Ecosystem Health and Management 3: 545-552.

Goulart, M. \& M. Callisto. 2003. Bioindicadores de qualidade de água como ferramenta em estudos de impacto ambiental. Revista da FAPAM 2 (1): 156-164.

Higuti, J. \& A.M. TAKETA. 2002. Spatial and temporal variation in densities of Chironomid larvae (diptera) in two lagoons and two tributaries of the upper Paraná River Floodplain, Brazil. Brazilian Journal of Biology 62 (4B): 807-818.

Hirabayashi K. \& R.S. Wotton. 1998. Organic matter processing by Chironomidae larvae (Diptera: Chironomidae). Hydrobiologia 382: 151-159.

Junk, W.J. \& C.J. DA Silva. 1995. Neotropical Floodplains: A comparison between the Pantanal of Mato Grosso and the 
large Amazonian River Floodplains, p. 195-217. In: J.G. TuNDISI; C.E.M. Bicudo \& T. Matsumura-Tundisi. (Eds). Limnology in Brazil. Rio de Janeiro, Brazilian academy of sciences, Brazilian Limnological Society, 376p.

JunK, W.J.; P.B. BAYley \& R.E. SPARKS. 1989. The flood pulse concert in river floodplain systems, p. 110-127 In: D.P. Dodge (Ed.). Proceeding of the international large Symposium (LARS). Ottawa, Canadian Special Publications of Fisheries and Aquatic, 106p.

Kikuchi, R.M. \& V.S. Uieda. 1998. Composição da comunidade de invertebrados de um ambiente lótico tropical e sua variação espacial e temporal, p. 157-173. In: J.L. Nessimian \& A.L. Carvalho (Eds). Ecologia de Insetos Aquáticos. Rio de Janeiro, PPGE-UFRJ, Série Oecologia brasiliensis, V+309p.

Krebs, C.J. 1989. Ecological methodology. Dordrecht, Harper \& Row Publishers, XI+654p.

Lencioni V. \& B. Rossano. 2005. Microdistribution of Chironomids (Diptera: Chironomidae) in Alpine streams: an autoecological perspective. Hydrobiologia 533: 61-76.

Li, A.; J. Frouz \& R.J. Lobinske. 2002. Spatio-temporal effects of selected physico-chemical variables of water, algae and sediment chemistry on the larval community of nuisance Chironomidae (Diptera) in a natural and a man-made lake in central Florida. Hydrobiologia 470: 181-193.

Marchese, M.R.; K.M. Watzen \& I. Ezcurra de Drago. 2005. Benthic invertebrate assemblages and species diversity patterns of the upper Paraguay River. River Research and Applications 21: 485-499.

Marques, M.M.G.S.M; F.A.R. Barbosa \& M. Callisto, 1999. Distribution and abundance of Chironomidae (Diptera, Insecta) in a impacted watershed in South-east Brazil. Revista Brasileira de Biologia 59: 553-561.

MerritT, R.W. \& K.W. Cummins. 1996. An introduction to the aquatic insects of north America. Kendall, Hunt Publishing, $3^{\text {rd }}$ ed., 861p.

Moreira S.S. \& J. Zunanon. 2002. Dieta de Retroculus lapidifer (Perciformes: Cichlidae), um peixe reofílico do rio Araguaia, estado do Tocantins, Brasil. Acta Amazônica 32 (4): 691705.

Moulton, T.P. 1998. Saúde integridade do ecossistema e o papel dos insetos aquáticos, p. 281-298. In: J.L. Nessimian \& A.L. Carvalho (Eds). Ecologia de insetos aquáticos. Rio de Janeiro, PPGE-UFRJ, Série Oecologia brasiliensis, V+309p.

Oliver, D.R. \& M.E. Dillon 1997. Chironomids (Diptera: Chironomidae) of the Yukon Arctic North Slope and Herschel Island, p. 615-635 In: Danks H.V. \& J.A. Downes (Eds). Insects of the Yukon. Ottawa, Biological Survey of Canada (Terrestrial Arthropods), 1034p.

Otт, A.P. \& G.S. Carvalho. 2001. Comunidade de Cigarrinhas (Hemiptera: Auchenorrhyncha) de uma Área de Campo do Município de Viamão, Rio Grande do Sul, Brasil. Neotropical
Entomology 30 (2): 233-243.

PсвAP. 1997. Plano de conservação da Bacia do Alto Paraguai - PCBAP. Brasília, Programa Nacional do Meio Ambiente, vol. 2, II+349p.

Príncipe, R.E. \& M.C. Corigliano. 2006. Benthic, drifting and marginal macroinvertebrate assemblages in a lowland river: temporal and spatial variations and size structure. Hydrobiologia 553: 303-317.

Robinson, C.T.; U. Uehlinger \& M. Hieber. 2001. Spatio-temporal variation in macroinvertebrate assemblages of glacial streams in the Swiss Alps. Freshwater Biology 46: 1663-1672.

Sanseverino, A.M.; J.L. Nessimian \& A.H.A. Oliveira. 1998. A fauna de Chironomidae (Diptera) em diferentes biótipos aquáticos na serra do Subaio (Teresópolis, RJ), p. 253-263. In: J.L. Nessimian \& A.L. Carvalho (Eds). Ecologia de insetos aquáticos. Rio de Janeiro, PPGE-UFRJ, Série Oecologia brasiliensis, $\mathrm{V}+309 \mathrm{p}$.

ScHMid, P.E. 1992. Population dynamics and resource utilization by larval Chironomidae (Diptera) in a backwater area of the River Danube. Freshwater biology 28: 111-127.

Serrano, M.A.S.; W. Severi, \& V.S.J. Toledo. 1998. Comunidade de Chironomidae e outros macroinvertebrados em um rio tropical de planície - Rio Bento Gomes/MT, p. 265-278. In: J.L. Nessimian \& A.L. Carvalho (Eds). Ecologia de insetos aquáticos. Rio de Janeiro, PPGE-UFRJ, Série Oecologia Brasiliensis, $\mathrm{V}+309 \mathrm{p}$.

Takeda, A.M.; G.Y. Shimizu; G.M. Shulz \& A.C.M. Silva. 1991. Zoobentos de quatro lagoas de várzea do alto rio Paraná (MS-Brasil), Influência do regime hidrológico sobre a comunidade. Revista UNIMAR 13 (2): 365-387.

TAKedA, A.M.; G.Y. Shimizu \& J. Higuti. 1997. Variação espaçotemporais da comunidade zoobentônica, p. 157-177. In: A.E.A.M Vazzoler; A.A. Agostinho \& N.S. Hahn. 1997. A planície de inundação do Alto rio Paraná: Aspectos físicos, biológicos e sócio-econômicos. Maringá, Editora da Universidade Estadual de Maringá, 460p.

Trivinho-Strixino, S. \& G. Strixino. 1995. Larvas de Chironomidae (Diptera) do Estado de São Paulo: guia de identificação e diagnose dos gêneros. São Carlos, Universidade Federal de São Carlos, 229p.

Trivinho-Strixino, S. \& G. Strixino. 2005. Chironomidae (Diptera) do rio Ribeira (divisa dos estados de São Paulo e Paraná) numa avaliação ambiental faunística. Entomología y Vectores 12 (2): 243-253.

WALKER, I. 1998. Population dymanics of Chironomidae (Diptera) in the central Amazon blackwater river Tarumã-Mirim (Amazonas, Brazil). In: J.L. Nessimian \& A.L. Carvalho (Eds). Ecologia de insetos aquáticos. Rio de Janeiro, PPGE-UFRJ, Série Oecologia Brasiliensis, V+309p.

Ward, J.V.; K. Tockner; D.B. Arscott \& C. Claret. 2002. Riverine landscape diversity. Freshwater Biology 47: 517-539.

Recebido em 03.V.2006; aceito em 04.VII.2007.

Revista Brasileira de Zoologia 24 (3): 565-572, setembro 2007 